\title{
Bodenmodelle zur Untersuchung des Schwingungsverhaltens bei Schmalgangstaplern
}

\author{
PROF. WiLlibald A. GÜNTHNER \\ LEHRSTUHL FÜR FÖRDERTECHNIK, MATERIALFLUSS, LOGISTIK (FML), TECHNISCHE UNIVERSITÄT MÜNCHEN \\ DR.-ING. JÜRGEN SCHMALZL \\ PRODUKTLINIE LAGER + SYSTEM, JUNGHEINRICH MOOSBURG GMBH
}

\section{Zusammenfassung}

Die Praxis zeigt, dass die aktuell gültigen Normen und Richtlinien für die Anforderungen an Böden in Schmalganglagern nicht dazu geeignet sind, die Laufruhe eines darauf betriebenen Schmalgangstaplers sicherzustellen. Durch eine Untersuchung der Fahrdynamik dieser Geräte mit Hilfe der Mehrkörpersimulation kann ein Zusammenhang zwischen den Bodenunebenheiten und dem Schwingungsverhalten des Staplers dargestellt werden. Hierfür sind alle wesentlichen, die Schwingungen beeinflussenden Baugruppen abzubilden, wobei ein besonderer Schwerpunkt auf die geeignete Abbildung des Bodens gelegt wird.

Mit periodischen und regellosen Unebenheiten werden zwei Gruppen von Unebenheiten unterschieden. Die systematische Behandlung der regellosen Unebenheiten ist nur unter Anwendung statistischer Methoden möglich. Man wählt den Weg über die Spektraldichtefunktionen und kann somit die Einflussgrößen auf die Bodengüte auf einen einzigen Parameter reduzieren. Zugleich eröffnet die Verwendung der Spektraldichtefunktionen die Anwendbarkeit eines Algorithmus zur Generierung von realitätsnahen Unebenheitsverläufen für die Simulation.

\section{Ausgangssituation und Problemstellung}

Die allgegenwärtige Forderung nach höherer Leistung fördertechnischer Geräte hat ihre Folgen auch für den Bereich der Schmalgangstapler. Die Hersteller dieser Geräte realisieren bezüglich Tragfähigkeit, Fahrgeschwindigkeit, Hubgeschwindigkeit und Fahrbeschleunigung bei neuen Modellen beträchtliche Verbesserungen.

Vor diesem Hintergrund tritt jedoch eine ungeeignete Bewertung der Bodenqualität für staplerbetriebene Lagerbereiche durch die gültige Norm vermehrt als Problem auf. So erweisen sich in einigen Fällen Böden, deren Toleranzen der hierfür anzuwendenden Norm DIN 15185 T1 voll entsprechen, teilweise als untauglich. In anderen Fällen wird dagegen der effiziente Betrieb des Staplers durch Bodenunebenheiten behindert, zu denen in der Norm keine Aussage getroffen wird (z. B. extrem kurzwellige Unebenheiten). Die Schleifbearbeitung des Bodens durch einen Bodensanierer führt zwar in vielen Fällen zur erwünschten Laufruhe des Staplers, jedoch ist diese Maßnahme mit hohen Kosten und teilweise langen Ausfallzeiten des Lagerbereichs verbunden, was die Betreiber vielfach von einer Sanierung abhält.

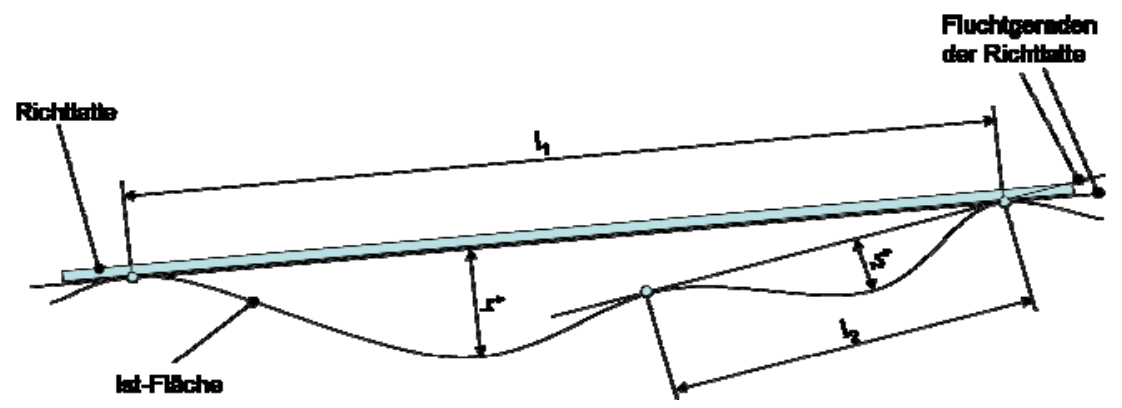

Abbildung 1: Definition der Ebenheitstoleranz ([DIN-18202], Bild 6) 
Die DIN 18202 „Toleranzen im Hochbau“ definiert den Begriff „zulässige Ebenheitstoleranz“ als „das Abmaß eines Tiefpunktes von der Verbindungslinie zweier benachbarter Hochpunkte“ oder „das Abmaß eines Hochpunktes von der Verbindungslinie zweier benachbarter Tiefpunkte“, wobei die Verbindungslinie nicht horizontal verlaufen muss (vgl. Abb. 1). Diese Definition hat den Vorteil, dass mit sehr einfachen Mitteln die Ebenheit eines Bodens nach Norm überprüft werden kann. Wie dem Titel der Norm zu entnehmen ist, bezieht sich diese auf alle Bereiche des Hochbaus und geht in ihrer Anwendung weit über den hier betrachteten Bereich der Lagertechnik hinaus.

Für den Betrieb von Schmalgangstaplern in Lagersystemen sind besondere, über die Grenzwerte der DIN 18202 hinaus gehende Anforderungen an den Boden zu stellen.

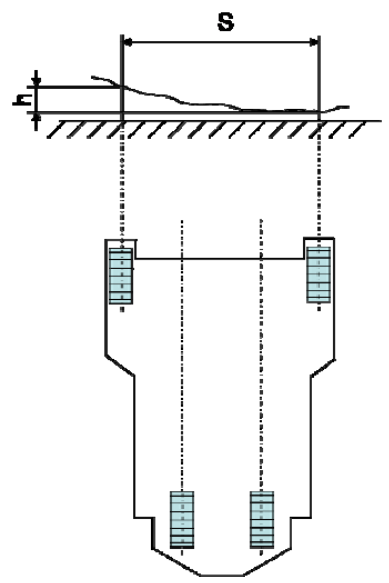

Abbildung 2: Definition des maximalen Höhenunterschieds quer zur Fahrtrichtung ([DIN-15185], Bild 1)

Diese wurden in der DIN 15185 „Lagersysteme mit leitliniengeführten Flurförderzeugen“ Teil 1 festgelegt. Unterschieden wird in Höhenunterschiede quer zur Fahrspur (Abb. 2) und Ebenheitstoleranzen längs den Fahrspuren. Die DIN 15185 T1 und damit die dort angegebenen Grenzwerte wurden im August 1991 veröffentlicht. Die Grenzwerte wurden ohne wissenschaftliche Grundlage festgesetzt. Vielmehr stellten sie einen Kompromiss zwischen der technisch realisierbaren Bodenqualität und der minimalen Anforderung hinsichtlich des Betriebs des Flurförderzeugs dar.

Außerhalb des Geltungsbereichs der DIN 15185 T1 sind folgende technische Regeln für die Bodenqualität zum Einsatz von Schmalgangstaplern interessant:

- $\quad$ TR34 (Großbritannien)

- $\quad$ ACI 117-90 (USA).

TR34 ist in diesem Zusammenhang kein Pendant zur DIN-Norm, sondern eine Art zur Norm erhobene „Firmenvorschrift“. Ihre Einhaltung muss aufgrund der relativ komplexen Formulierung mit speziellen messtechnischen Geräten überprüft werden. Hinsichtlich der Anforderungen an den Boden stellt sich die TR34 unwesentlich genauer als die DIN 15185 T1 dar. Dennoch fehlen auch hier Hinweise, wie Bodenprofile hinsichtlich der Welligkeit klassifiziert werden können.

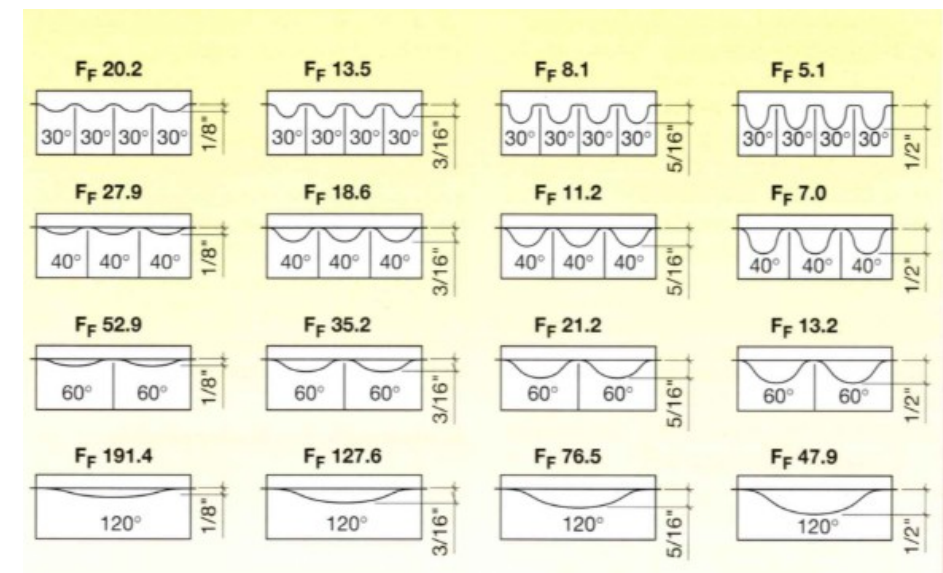

Abbildung 3: Klassifikation von Bodenprofilen aufgrund der Welligkeit nach [ACI-117], [Günthner2005] 
Die Klassifikation der Bodenprofile nach ACI 117-90 basiert auf einem Kennzahlensystem, den sog. FNummern ( $F_{F}$ für „floor flatness“ - Ebenheitskennzahl, vgl. Abb. 3). Die Kennzahlen erfassen auch die Welligkeit des Bodens. Anzunehmen ist, dass hier eine bessere Korrelation zwischen Bodenklassifizierung und Schwingungsverhalten des Flurförderzeugs als bei der DIN 15185 T1 und dem TR34 besteht.

Um ggf. für eine Überarbeitung der DIN bzw. zur Vereinheitlichung der europäischen Normung eine wissenschaftliche Grundlage zu schaffen, ist es erforderlich, die Zusammenhänge zwischen der Unebenheit des befahrenen Bodens und dem dynamischen Verhalten des darauf betriebenen Flurförderzeugs zu ermitteln. Zu finden ist folglich eine neue Systematik und Prüfmethode, die den ursächlichen Zusammenhang zwischen Boden und Staplerschwingung besser widerspiegelt. Ziel soll es aber nicht sein, lediglich die Grenzwerte der aktuellen DIN $15185 \mathrm{~T} 1$ an die Bedürfnisse neuer Gerätegenerationen anzupassen. Die dynamischen Daten des Flurförderzeugs müssen in diesem System wieder zu finden sein. Dadurch lässt sich neben dem sicheren Betrieb des Fahrzeugs auch gewährleisten, dass keine unnötig hohen Anforderungen an die Bodenebenheit gestellt werden.

Für eine dafür angesetzte Untersuchung müssen neben den dynamischen Größen auch die wichtigen Einflussparameter, wie Hubhöhe, Last, Elastizität des Hubgerüsts, Spiel in den Führungen des teleskopierbaren Hubgerüsts und die Radelastizität, mit berücksichtigt werden. In der Vergangenheit gab es bereits einige Ansätze zur messtechnischen Untersuchung dieser Parametereinflüsse. Die hohe Anzahl an Parametern führte jedoch zu sehr umfangreichen Messreihen. Aufgrund dieser großen Datenmenge gestaltete sich die Auswertung schwierig, so dass nicht im erwarteten Umfang Erkenntnisse gewonnen werden konnten. Daher wird eine modellhafte Untersuchung angestrebt. Wegen der zu erwartenden hohen Komplexität des Modells sowie der Vielzahl an zu variierenden Parametern empfiehlt sich die Zuhilfenahme rechnergestützter Simulationswerkzeuge, wie Mehrkörper- und Finite-Elemente-Simulationen. Solche Tools haben sich in den letzten Jahren - nicht zuletzt aufgrund der extremen Zunahme an Rechenkapazitäten der Hardware - zu leistungsfähigen Softwarepaketen entwickelt, die äußerst realitätsnahe und sehr genaue Modelluntersuchungen ermöglichen. Im vorliegenden Fall des Schmalgangstaplers wird das Simulations-Paket MSC.ADAMS 2003 für eine Mehrkörpersystem-Simulation eingesetzt. Die Abbildung als Rechnermodell bezieht sich auf die relevanten Schwingungsformen des Hubgerüsts. Um die im System vorhandenen Elastizitäten in der Untersuchung im erforderlichen Maß zu berücksichtigen, ist die Erstellung eines hybriden Mehrkörpermodells notwendig. Entscheidenden Einfluss auf das Systemverhalten haben die Komponenten Hubhydraulik, Bereifung sowie das Hubgerüst aufgrund deren Elastizitäten. Daher ist den Abbildungen dieser Komponenten besondere Aufmerksamkeit zu widmen.

\section{Mehrkörpermodell}

Unter Verwendung der Simulationssoftware MSC.ADAMS 2003 entstand am Lehrstuhl fml der TU München ein Rechnermodell des Systems Flurförderzeug-Boden. Schwerpunkte bei der Abbildung der einzelnen Baugruppen des Geräts stellten die Teilmodelle für die Räder, die Hubhydraulik, die spielbehafteten Führungen sowie das Hubgerüst mit seiner Elastizität dar. Letzterer Aspekt führte zu einem so genannten hybriden Mehrkörpersystem. Ein solches Modell ermöglicht eine sehr realitätsnahe Abbildung in Vergleich zu herkömmlichen Mehrkörpermodellen, da es neben starre auch elastische Körper zulässt. So kann für jeden Einzelkörper entschieden werden, ob es in guter Näherung eine starre Modellierung ausreichend ist, oder ob eine elastische Modellierung, wie im vorliegenden Fall das Hubgerüst, notwendig wird. Durch eine Reduktion der hohen Zahl an Freiheitsgraden, wie sie in einem elastischen Modell - beispielsweise ein FE-Modell vorkommen, auf eine für die Mehrkörpersimulation angemessene Zahl kann ohne Genauigkeitsverlust das elastische Verhalten auch in der Mehrkörpersimulation bei akzeptablen Rechenzeiten mit berücksichtigt werden. 


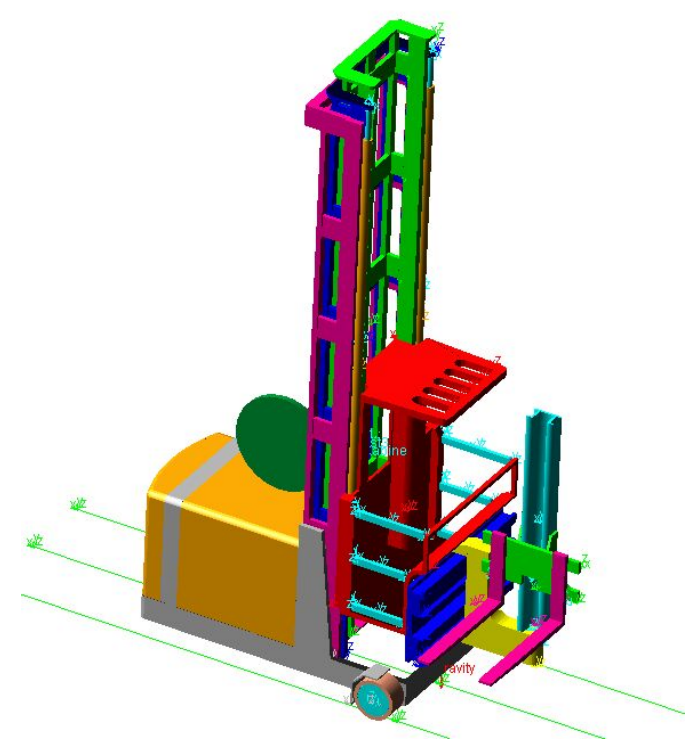

Abbildung 4: Mehrkörpermodell eines Schmalgangstaplers

Für eine effiziente Untersuchung und praktikable Rechenzeiten bei der Simulation muss das System StaplerBoden auf die schwingungstechnisch relevanten Modellkomponenten abstrahiert werden. Als wesentliche Schwingungskomponenten sind zu identifizieren:

Tabelle 1: Schwingungsformen und ihre Ursachen

\begin{tabular}{|l|l|}
\hline Schwingungsform & beeinflusst durch \\
\hline Hubschwingung & Hubhydraulik, Hubketten, Vulkollanräder, Rahmen \\
\hline Torsionsschwingung & Hubmast, Exzentrizität der Last \\
\hline Querschwingung & $\begin{array}{l}\text { Hubmast, Führungsspiel, Hubhydraulik, Hubketten, } \\
\text { Rahmen, Vulkollanräder }\end{array}$ \\
\hline Längsschwingung & Hubmast, Führungsspiel, Rahmen, Vulkollanräder \\
\hline
\end{tabular}

Aufgrund der höheren Komplexität wird das Modell in die Teilmodelle zerlegt:

- Hubgerüst

- Fahrwerk

- Boden

\subsection{Bodenmodellierung}

Aufgrund der hohen Anforderung an die Dynamik von Kraftfahrzeugen sowie an die Schwingungsbelastung des Fahrers (Fahrkomfort) werden in der Fahrzeugtechnik in umfangreichem Maße Untersuchungen vorgenommen. Diese erfordern auch eine adäquate Abbildung der Anregung, also der Unebenheiten der Fahrbahn. Hinsichtlich der Unebenheiten längs der Fahrspur hat sich eine standardisierte Formulierungsweise etabliert. Grundsätzlich ist für den vorliegenden Fall hinsichtlich der Art der Unebenheiten die Unterscheidung von zwei Anteilen empfehlenswert:

- $\quad$ regelloser Anteil; stochastische Störung

- $\quad$ periodische Unebenheiten; deterministische Störung 


\subsubsection{Regelloser Anteil}

Die Abbildung regelloser Unebenheiten erfordert zum einen die mathematische Formulierung der Unebenheiten sowie eine Bewertung der Charakteristik und Qualität des jeweils abgebildeten Bodens.

Dabei nutzt man die Grundaussage der Fourier-Analysis, dass jegliche Funktion angenähert durch eine Summe aus harmonisch verwandten Sinus- und Cosinusfunktionen darstellbar ist. Im vorliegenden Fall geht man davon aus, dass gleichzeitig Anteile aller Wellenlängen im regellosen Verlauf des Bodenprofils enthalten sind. Zur Datenreduktion und hinsichtlich einer Weiterverarbeitung der Fahrbahnunebenheiten zu Schwingungsgrößen ist die Darstellung der Unebenheiten mit Hilfe der spektralen Dichte vorteilhaft. [Popp1993], [Kortüm1994]

Wird der Verlauf der Unebenheiten eines Bodens als Funktion mit Hilfe der Fourier-Analyse dargestellt, so beschreibt die spektrale Dichte die Dichteverteilung der Frequenzen der zur Darstellung verwendeten harmonischen Schwingungsfunktionen. In diesem Zusammenhang werden auch die Begriffe "farbiges" und "weißes Rauschen" verwendet, die eine anschauliche Deutung der spektralen Dichte bieten.

\subsubsection{Farbiges Rauschen (Abb. 5):}

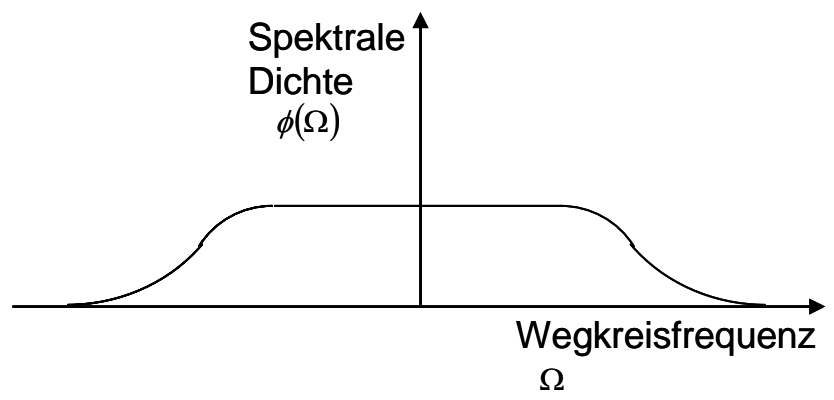

Abbildung 5: Verlauf der spektralen Dichte bei "farbigem Rauschen"

Die spektrale Dichte beim "farbigen Rauschen" ist über ein relativ breites Band von Frequenzen konstant. Außerhalb dieses Bereichs fallen die Werte für die spektrale Dichtefunktion stark ab. Dieser Verlauf ist mit der Farbenlehre in der Optik entsprechend interpretierbar: bei farbigem Licht herrschen ebenfalls Frequenzen eines für die jeweilige Farbe charakteristischen Wellenlängenbereichs des Lichts vor.

\subsubsection{Weißes Rauschen (Abb. 6):}

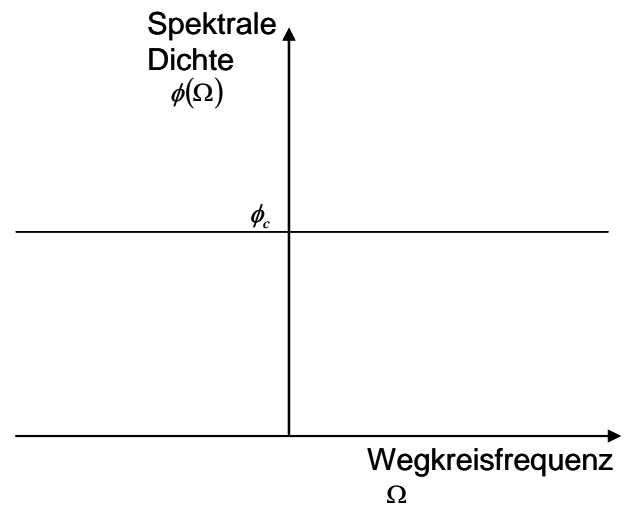

Abbildung 6: Verlauf der spektralen Dichte bei "weißem Rauschen"

Dagegen stellt das "weiße Rauschen" einen Grenzfall des "farbigen Rauschens" dar. Das oben angesprochene Frequenzband ist dabei theoretisch unendlich groß, d.h. alle Frequenzen kommen vor. Die Farbenlehre deutet das gleichzeitige Auftreten aller Wellenlängen des Lichts als weißes Licht. 
Aus Erfahrungen mit Messungen von Unebenheiten bei Straßen haben sich standardisierte Straßenspektren ergeben [Braun1991]. Die relativ einfache Formulierung ermöglicht die Standardisierung und die Reduzierung auf wenige Einflussparameter.

Bei Messungen von Unebenheiten bei Straßen [Schuknecht91] ergab sich, dass die spektrale Dichte $\phi_{h}(\Omega)$ folgendermaßen angegeben werden kann:

$\phi_{h}(\Omega)=\phi_{h}\left(\Omega_{0}\right) \cdot\left(\frac{\Omega}{\Omega_{0}}\right)^{-w}$

mit:

$\Omega_{0}=1 \frac{1}{m} ; \quad$ Bezugs-Wegkreisfrequenz bei der Wellenlänge $L_{0}=2 \pi \mathrm{m}=6,28 \mathrm{~m}$

$\phi_{h}\left(\Omega_{0}\right)$; $\quad$ Unebenheitsmaß; große Werte bedeuten unebenere Oberfläche

w; $\quad$ Welligkeit, große Werte bedeuten bevorzugt lange Wellen

Im doppeltlogarithmischen Koordinatensystem erscheint obige Gleichung als Gerade. Die Lage wird dabei vom Unebenheitsmaß $\phi_{h}\left(\Omega_{0}\right)$ festgelegt, die Steigung der Geraden repräsentiert die Welligkeit $w$.

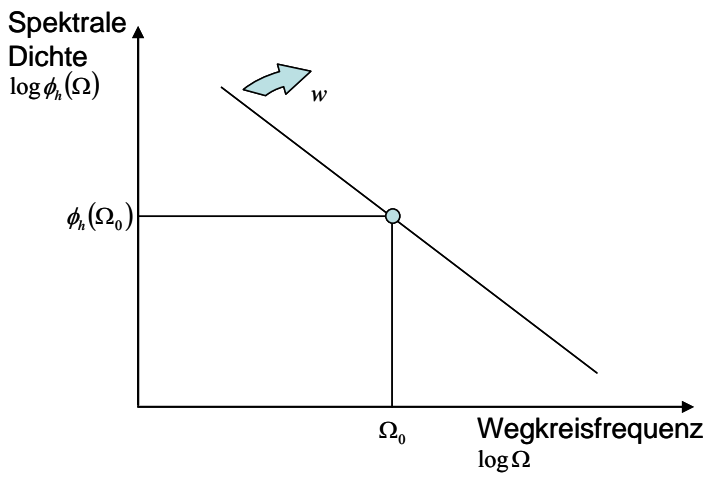

Abbildung 7: Doppeltlogarithmische Darstellung der spektralen Dichte über die Wegkreisfrequenz

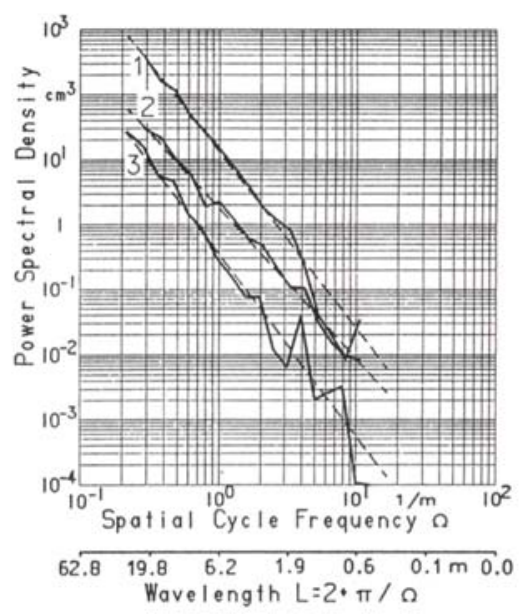

Abbildung 8: Messprotokolle deutscher Fahrstraßen, doppeltlogarithmische Darstellung der spektralen Unebenheitsdichte [Schuknecht91]

So wird es möglich, den Ebenheitszustand einer Fahrbahn durch eine Gerade zu charakterisieren. In Abbildung 8 sind die Messprotokolle dreier vermessener Fahrstraßen dargestellt. Man erkennt, dass die Messpunkte aller drei Strecken in guter Näherung als Trendlinie eine Gerade besitzen. 
Sie unterscheiden sich lediglich im Achsenabschnitt, die Steigungen der Geraden liegen im selben Größenordnungsbereich. Durch mathematische Umformung der Grundgleichung der spektralen Dichte lässt sich zeigen, dass für $w=2$ die Spektraldichte dem "weißen Rauschen" entspricht. Die plausible Erklärung liegt darin, dass bei regellosen Bodenunebenheiten alle Wellenlängen mit gleicher Häufigkeit vertreten sind. In der Literatur [Schuknecht91] wird als stochastische Anregungsfunktion für Fahrbahnen daher ebenfalls "weißes Rauschen" empfohlen. Zudem deckt sich dies mit den Beobachtungen an vermessenen Fahrbahnen, bei denen $W$ stets im Größenordnungsbereich von $2( \pm 10 \%)$ liegt.

Somit kann unter der Verwendung der spektralen Dichte der Zustand eines regellosen Bodenprofils prinzipiell mit lediglich einem Parameter beschrieben werden, nämlich dem Unebenheitsmaß $\phi_{h}\left(\Omega_{0}\right)$, oder auch U:

Weitere Messungen [Mitschke1989] ergaben, dass sich Fahrstraßen im Bereich von U=18 (sehr schlecht) bis $\mathrm{U}=0,3$ (sehr gut) bewegen. Hinsichtlich der qualitativ besseren Böden in Lagern wurde für die Untersuchung ein Bereich unterhalb $\mathrm{U}=1$ angestrebt. Die Einordnung der dabei entstehenden Böden nach DIN 15185 T1 bestätigt dies.

\subsubsection{Sinusförmiger Anteil}

Als zweite Anregungsart soll im Untersuchungsprogramm die Schwingungsanregung durch periodische Unebenheiten vorgesehen werden. Die im vorangegangenen Kapitel dargestellten Bodenprofile werden nun durch einfache Sinusfunktionen ersetzt. Die hier möglichen Variationsmöglichkeiten Amplitude $\hat{h}$ und Wellenlänge $L$ gehen ohne mathematische Umwege in die Funktion ein:

$h(x)=h_{0}+\hat{h} \sin \left(\frac{2 \pi}{L} x-\alpha\right)$

Im Rahmen der Untersuchung wird anfänglich ein Boden mit einer Wellenlänge $L=0,5 m$ und einer Amplitude $\hat{h}=1 \mathrm{~mm}$ hinterlegt. Zur Untersuchung des Worst-Case werden linke und rechte Fahrspur $\left(h_{l}(x)\right.$ bzw. $h_{r}(x)$ ) gegengleich ausgeführt:

$\alpha_{l}=\alpha_{r}+\pi$

So steht einem Wellenberg links immer ein Wellental rechts gegenüber und umgekehrt. Es ist anzumerken, dass dieser Boden theoretisch nach DIN 15185 T1 alle Grenzwerte in Längs- und Querrichtung einhält. Durch Variation der Fahrgeschwindigkeit, bzw. Einstellung der kritischen Geschwindigkeit $v_{\text {Eigen }}$ kann die Anfälligkeit des Geräts gegen Anregung mit Eigenfrequenz $f_{\text {Eigen }}$ geprüft werden:

$v_{\text {Eigen }}=L f_{\text {Eigen }}$

Zudem liefert eine Fahrt mit geringer Beschleunigung, bei der sämtliche Geschwindigkeits- und damit auch alle Anregungsfrequenzbereiche durchlaufen werden, eine Aussage darüber, ob nur die Endgeschwindigkeit für die Laufruhe bestimmend ist, oder ob schon kurzzeitige Anregungen mit Eigenfrequenz Auswirkungen haben.

\subsubsection{Rechnergestützte Erzeugung von Bodenprofilen}

Zur Untersuchung des Schwingungsverhaltens, zu dem die Bodenunebenheiten das bewegte Modell anregen, muss für die Simulation das durch das Unebenheitsmaß und die Welligkeit charakterisierte Bodenprofil in einen konkreten Höhenverlauf umgewandelt werden. Da es sich bei regellosen Unebenheiten um einen skalaren stochastischen Prozess handelt, können aus den beiden Parametern Realisierungen des stochastischen Prozesses abgeleitet werden. Man geht dabei den umgekehrten Weg wie bei der Herleitung der spektralen Dichte und nutzt wiederum die Theorie, dass der Gesamtverlauf des Höhenprofils durch eine (endlose) Reihe harmonischer Funktionen erzeugt werden kann [Schuknecht91]. Die spektrale Dichte stellt hinsichtlich der Frequenzen eine kontinuierliche Funktion dar, d.h. es müssten alle Frequenzen von 0 bis $\infty$ in infinitesimal kleinen Abständen herangezogen werden. In der Praxis müssen durch eine Diskretisierung eine überschaubare Anzahl von Frequenzen in einem sinnvollen Bereich zur Generierung des Bodenprofils herangezogen werden. Da zudem eine Beschränkung auf einen Frequenzbereich erfolgt, legt man genau genommen nun nicht mehr das Frequenzspektrum des weißen Rauschens zugrunde, sondern man nähert das weiße Rauschen durch ein adäquates farbiges Rauschen an. Die Ermittlung des relevanten Frequenzbereichs aus der Eigenfrequenzanalyse des Modells stellt sicher, dass durch die Näherung keine maßgeblichen Anregungsformen vernachlässigt werden. 
Allgemein hat ein Höhenprofil, das aus einer Addition von Sinus-Funktionen im Sinne der Fourieranalyse hervorgeht, folgende Form:

$$
h(x)=h_{0}+\sum_{n=1}^{N} \hat{h}_{n} \sin \left(\Omega_{n} x-\alpha_{n}\right)
$$

mit

$h_{0} \quad$ „Nulllage“ des Höhenprofils

$\Omega_{n} \quad$ Wegkreisfrequenz der n-ten Sinusfunktion

$\hat{h}_{n} \quad$ zugehörige Amplitude der n-ten Sinusfunktion

$\alpha_{n} \quad$ Phasenlage der n-ten Sinusfunktion

Die erforderliche Diskretisierung legt die Anzahl $N$ der summierten Sinusfunktionen fest. Man verwendet Wegkreisfrequenzbänder, innerhalb denen die spektrale Unebenheitsdichte in guter Näherung als konstant angenommen werden kann. Die in der doppeltlogarithmischen Darstellung als Gerade auftretende Unebenheitsdichte wird dadurch durch eine Treppenfunktion angenähert (Abb. 9).

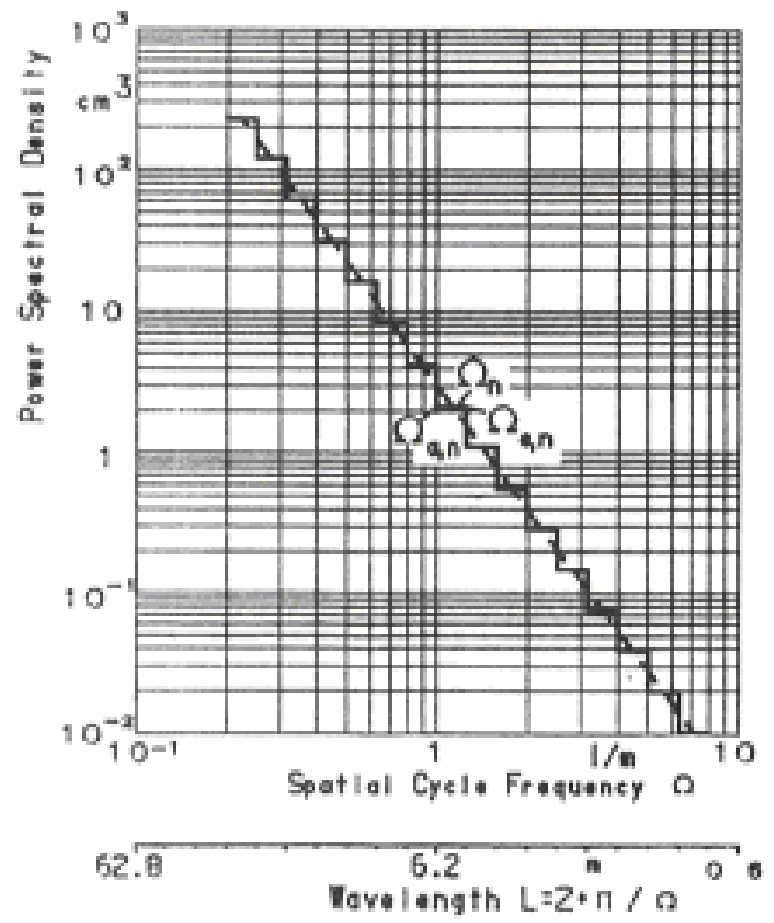

Abbildung 9: Diskretisierung der spektralen Dichte [Schuknecht91]

Die Stufenbreite ist in der Darstellung konstant, so dass für die Grenzen $\Omega_{a, n}$ und $\Omega_{e, n}$ der Bänder gilt:

$b=\frac{\Omega_{e, n}}{\Omega_{a, n}}=$ const.

Das Verhältnis b wird als Bandfaktor bezeichnet. Nähert sich b dem Wert 1, so erhält man eine feinere Stufung und schmalere Bandbreiten. In der Literatur [Mitschke1989], [Schuknecht91] wird für b=1,1 empfohlen. Der Mittelwert eines Bandes $\Omega_{n}$ ergibt sich aus dem geometrischen Mittel der Grenzen:

$\log \Omega_{n}=\frac{\log \Omega_{a, n}+\log \Omega_{b, n}}{2}$

Für das nächste Band gilt:

$\Omega_{a, n+1}=\Omega_{e, n}$ 
Jedem Band wird in Abhängigkeit des zugehörigen Mittelwerts eine Amplitude für die zur Addition herangezogene Sinusfunktion ermittelt. Die Berechnungsvorschrift ergibt sich aus dem Integral für den Effektivwert der Unebenheitshöhe mit der diskretisierten Unebenheitsdichte:

$h_{e f f, n}^{2}=\int_{\Omega_{a, n}}^{\Omega_{e, n}} \phi_{h}(\Omega) d \Omega$

mit $\hat{h}_{n}=\sqrt{2} h_{\text {eff }, n}$ ergibt sich für die Amplitude:

$\hat{h}_{n}\left(\Omega_{n}\right)=\sqrt{2\left(\phi_{h}\left(\Omega_{0}\right) \cdot\left(\frac{\Omega_{n}}{\Omega_{0}}\right)^{-w} \cdot\left(\sqrt{b}-\frac{1}{\sqrt{b}}\right) \cdot \Omega_{n}\right)}$

Der Wert $\Omega_{0}$ stellt die Bezugswegkreisfrequenz dar und wird vereinbarungsgemäß mit $\Omega_{0}=1 \frac{1}{\mathrm{~m}}$ angesetzt.

Das Unebenheitsmaß wird für diese Bezugswegkreisfrequenz angegeben.

Für die Darstellung des weißen Rauschens ist es wie erwähnt theoretisch erforderlich, einen unendlich großen Frequenzbereich abzubilden. Für die praktische Handhabbarkeit beschränkt man sich auf einen endlichen Frequenzbereich, der sich an den physikalischen Erfordernissen der Untersuchung orientiert. Durch eine Eigenfrequenzanalyse am Modell werden die relevanten Zeitfrequenzbereiche hinsichtlich der Anregung ermittelt. Der in der Fahrzeugtechnik häufig gewählte Frequenzbereich von 1 bis $25 \mathrm{~Hz}$ ist auch für den vorliegenden Fall gut anwendbar, da er alle relevanten Eigenfrequenzen des Geräts abdeckt. Unter Berücksichtigung der minimalen und maximalen Fahrgeschwindigkeiten, die untersucht werden, ergibt sich daraus ein Wellenlängenbereich:

$$
\begin{aligned}
& L_{\text {min }}=\frac{v_{\text {min }}}{f_{\text {max }}}=\frac{0,5 \frac{\mathrm{m}}{\mathrm{s}}}{25 \mathrm{~Hz}}=0,02 \mathrm{~m} ; \\
& L_{\text {max }}=\frac{v_{\max }}{f_{\text {min }}}=\frac{3 \frac{\mathrm{s}}{\mathrm{s}}}{1 \mathrm{~Hz}}=3 \mathrm{~m} .
\end{aligned}
$$

Einfluss auf ein mit beschriebenem Algorithmus erzeugtes Bodenprofil haben somit folgende Faktoren:

- $\quad \max . / \mathrm{min}$. Geschwindigkeit des Geräts bei der Simulation

- $\quad$ max./min. berücksichtigte Eigenfrequenz des Geräts

- Bandfaktor b

- Unebenheitsmaß $\phi_{h}\left(\Omega_{0}\right)$, im Weiteren vereinfachend als Unebenheitsmaß U bezeichnet

- Welligkeit $w$, für regellose Böden $w=2$ [Braun1991]

Unter Einsatz eines Rechnertools, in dem obige Zusammenhänge hinterlegt sind und das die vorgenannten Eingabeparameter enthält, lassen sich nun auf einfache Art und Weise beliebig viele Bodenprofile zur strukturierten Simulationsdurchführung erzeugen. Das Tool errechnet die Wellenlängenbereiche, ermittelt mit dem Bandfaktor die zu addierenden Wegkreisfrequenzen, bestimmt nach angegebener Gleichung die zugehörigen Amplituden und führt die Addition der Sinusfunktionen für jeden Punkt des Bodenprofils durch, nachdem für jede Funktion eine Phasenlage per Zufallsgenerator zugeordnet wurde. Schließlich gibt es das Bodenprofil in Form von Koordinaten in einer für die Mehrkörpersimulation verarbeitbaren Formatierung aus.

MSC.ADAMS liest diese Koordinaten als Matrix mit drei Spalten (Raumkoordinaten) und einer der Anzahl der Werte entsprechenden Zeilenzahl ein.

\section{Simulationsergebnisse}

\subsection{Einfluss der Bodenunebenheiten}

Neben der Gewinnung der bislang geschilderten Erkenntnisse, die sich teilweise schon während der Modellbildungsphase ergeben, indem Teilmodelle überprüft und die entsprechenden Parameter variiert werden, wird für den Hauptverwendungszweck des Modells, nämlich der Darstellung des Einflusses der Bodenunebenheiten, eine systematische Untersuchung nach Fertigstellung und Validierung des Modells durchgeführt. 


\subsubsection{Regellose Unebenheiten}

Unter Verwendung der dargestellten Systematik zur Abbildung von Bodenunebenheiten wurden zahlreiche Simulationsläufe mit qualitativ unterschiedlichen Böden vorgenommen. Variiert wurde der Parameter "Unebenheitsmaß" $\phi_{h}\left(\Omega_{0}\right)$, vereinfachend auch als "U" bezeichnet.

Modellkonfiguration:

- Schmalgangstapler mit Dreifach-Hubgerüst, max. Hubhöhe $12500 \mathrm{~mm}$

- Last: $1000 \mathrm{~kg}$

- Hubhöhe: $10 \mathrm{~m}$

- Spurweite $1450 \mathrm{~mm}$

- Fahrgeschwindigkeit: $10,5 \mathrm{~km} / \mathrm{h}$

Untersuchte Böden:

\begin{tabular}{|c|c|c|c|c|c|}
\hline 0,1 & 0,4 & 0,5 & 0,55 & 0,6 & 0,7 \\
\hline
\end{tabular}

Als wesentliche Schwingungskomponente für den sicheren Betrieb eines Schmalgangstaplers wird im weiteren Verlauf die Querschwingung herangezogen. Zwischen den Regalen und dem Flurförderzeug ist ein Mindestabstand von 90mm einzuhalten. Eine kleine Schwingungsamplitude ist deshalb aufgrund der möglichen Regalkollision von entscheidender Bedeutung.

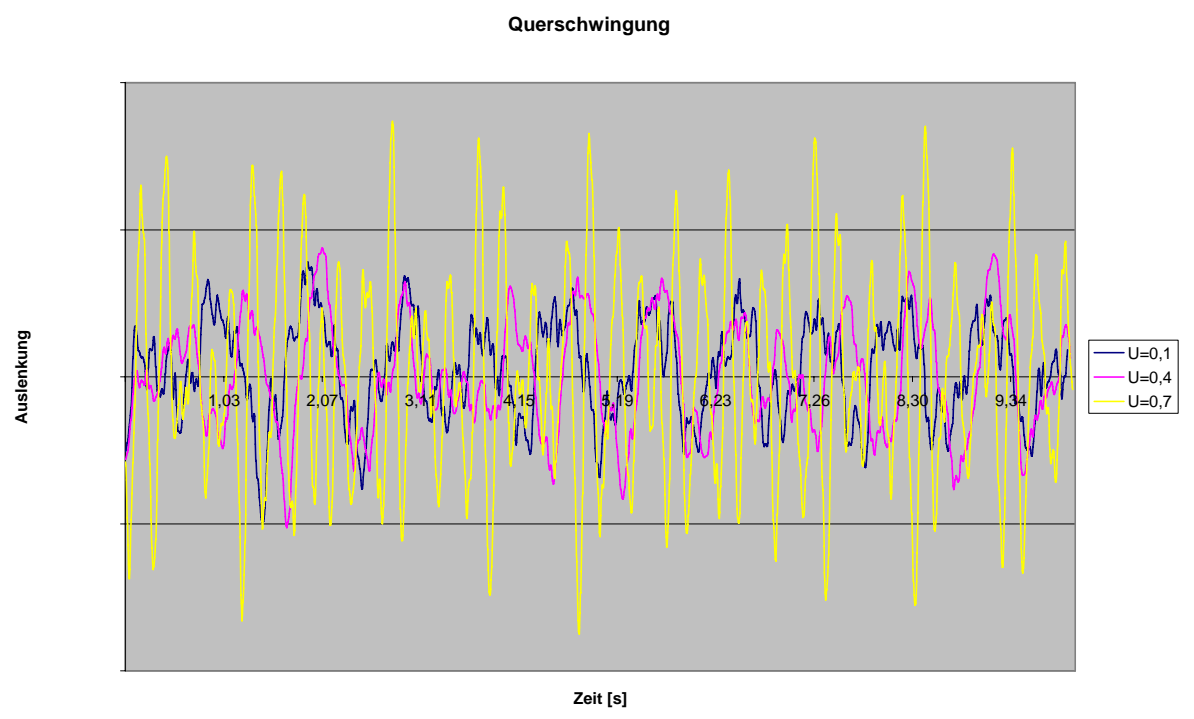

Abbildung 10: Querschwingungsamplitude bei unterschiedlichen Böden ( $U=0,1, U=0,4, U=0,7)$

Die zur groben Richtungsbestimmung untersuchten ersten Böden $U=0,1, U=0,4, U=0,7$ ergaben einen starken Anstieg der Querschwingungsamplitude zwischen den beiden letztgenannten Unebenheitsmaßen. Aus diesem Grund wurde verfeinernd eine Untersuchung von Böden mit $U=0,5, U=0,55, U=0,6$ nachgeschoben.

Aus Gründen der Geheimhaltung werden in der weiteren Darstellung die maximalen Querauslenkungen nicht als Absolutgrößen angegeben, sondern es wird eine parametrisierte Form gewählt. Dabei wird der Parameter d als maximal auftretende Querschwingungsamplitude bei einer Bodenunebenheit von $U=0,1$ festgesetzt. Die weiterhin dargestellten Auslenkungsgrößen werden als ein Vielfaches von d angegeben. 
Tabelle 2: Maximale Querauslenkung in Abhängigkeit der Bodenunebenheit

\begin{tabular}{|l|l|}
\hline U & Max. Querauslenkung \\
\hline 0,1 & $\boldsymbol{d}$ \\
\hline 0,4 & $\mathbf{1 , 5 4 d}$ \\
\hline 0,5 & $\mathbf{1 , 6 1 d}$ \\
\hline 0,55 & $\mathbf{1 , 9 6 d}$ \\
\hline 0,6 & $\mathbf{1 , 9 7 d}$ \\
\hline 0,7 & $\mathbf{2 , 5 5 d}$ \\
\hline
\end{tabular}

Der Tabelle 2 ist zu entnehmen, dass die Schwingungsamplitude bei zunehmender Bodenunebenheit (zunehmendes U) ansteigt.

Als Resultat kann deshalb festgehalten werden:

- Der Parameter U kann als Gradmesser sowohl für die Bodengüte bei Böden mit regellosen Unebenheiten als auch für die zu erwartende Laufruhe des Geräts verwendet werden. Dies ermöglicht eine Einordnung der simulierten Böden nach den aktuell anzuwendenden Normen und Richtlinien und liefert somit eine "Grenze" in Abhängigkeit von U, bei der ein Bodenprofil laut der jeweiligen technischen Regel tauglich ist oder nicht. Umgekehrt lassen sich dadurch auch die technischen Regeln untereinander vergleichen. Die Regel mit der niedrigeren "Grenze" im Hinblick auf den Parameter U kann als die "schärfere" Bedingung erachtet werden.

- Insgesamt sind die Amplituden quer zur Fahrtrichtung relativ klein. Eine Kollision mit den Regaleinrichtungen ist für die untersuchte Konfiguration auch bei schlechten Bodenqualitäten nicht zu erwarten.

\subsubsection{Periodische Unebenheiten}

Diese zweite häufig anzutreffende Form der Unebenheit wird unabhängig von den regellosen Unebenheiten betrachtet, auch wenn die gängigen Normen und Richtlinien hier keine Unterschiede machen.

Ein Bodenprofil, das unter Einhaltung der DIN 15185 T1 hinsichtlich der beiden äußeren Fahrspuren je ein Maximum der linken Sinusfunktion einem Minimum der rechten Sinusfunktion gegenüberstellt, wird mit unterschiedlichen Fahrgeschwindigkeiten durchfahren. Bei einer ersten Simulation mit $3 \mathrm{~m} / \mathrm{s}$ Fahrgeschwindigkeit (wie bei den Untersuchungen zu den regellosen Böden) ergibt sich bereits eine relativ hohe Querschwingungsamplitude.

Ersetzt man die konstante Fahrgeschwindigkeit durch einen linear ansteigenden Geschwindigkeitsverlauf, so werden während der Simulationszeit alle Geschwindigkeitsbereiche durchlaufen (Geschwindigkeit innerhalb 30s Simulationszeit von $1 \mathrm{~m} / \mathrm{s}$ auf $3 \mathrm{~m} / \mathrm{s}$ zunehmend). Da die Geschwindigkeit moderat gesteigert wird, sind Effekte aus der Beschleunigung zu vernachlässigen.

Anmerkung: Auch hier werden die Amplituden als Vielfaches von d (= max. Querauslenkung bei $U=0,1)$, angegeben.

Tabelle 3: Vergleich periodische - regellose Anregung

\begin{tabular}{|l|l|}
\hline Versuch & Max. Querauslenkung \\
\hline konst. $3 \mathrm{~m} / \mathrm{s}$ & $\mathbf{2 , 4 7 \boldsymbol { d }}$ \\
\hline von $1 \mathrm{~m} / \mathrm{s}$ auf $3 \mathrm{~m} / \mathrm{s}$ beschleunigt & $\mathbf{3 , 3 3 d}$ \\
\hline vgl.: regelloser Boden, $U=0,7$ & $\mathbf{1 , 9 7 d}$ \\
\hline
\end{tabular}


Es ist zu erkennen, dass auch schlechte regellose Böden geringere Schwingungsamplituden hervorrufen als periodische Böden. Erreicht man im Rahmen von Beschleunigungsvorgängen kurze Zeitbereiche, in denen die Periodizität des Bodens Resonanz im Gerät hervorruft, steigt die Amplitude auf das Doppelte im Vergleich zu der auf schlechtem regellosem Boden. Da die Querschwingungsamplituden erst bei Resonanz in kritischen Bereichen für Regalkollisionen liegen, bei völlig regellosen Böden jedoch weit darunter, ist anzunehmen, dass nicht die absoluten Unebenheiten eines Bodens eine Rolle bei der Schwingungsanfälligkeit des Staplers spielen, sondern dass in allen Fällen von unruhigem Lauf in der Praxis nur eine Periodizität des Bodens als Ursache in Frage kommt.

Hieraus ergibt sich eine wertvolle Erkenntnis für die Neufassung technischer Regel zur Bewertung von Bodenunebenheiten. Die ausschließliche Definition einer festen Grenze für die Tauglichkeit von Böden zum Betrieb von Schmalgangstaplern ist nicht Ziel führend. Vielmehr ist auf die Form der Bodenunebenheit zu achten. Es ist ein Verfahren zu finden, dass sowohl die periodischen als auch die regellosen Unebenheiten entsprechend gewichtet und wertet. Ähnlich wie bei der Formulierung der Anregung im Modell ist auch hier eine Betrachtung mit statistischen Mitteln viel versprechend.

\section{Zusammenfassung und Ausblick}

Somit kann hinsichtlich der Eignung von Böden im Lager für den Betrieb von Flurförderzeugen zusammengefasst werden:

- Das Unebenheitsmaß stellt das ideale Bewertungskriterium für regellose Böden dar.

- Die Welligkeit muss in Abhängigkeit der Amplitude und der Wellenlänge in die Bewertung mit einfließen.

An eine Richtlinie zur Bewertung der Eignung eines Bodens werden daraus folgende Anforderungen abgeleitet:

- Das Bewertungsschema muss mit dem Unebenheitsmaß korrelieren.

- Die Welligkeit muss mit einfließen.

- Die messtechnische und mathematische Überprüfung muss einfach möglich sein.

Eine nach obigen Kriterien brauchbare Formulierung des Bewertungsschemas lässt sich unter Verwendung der Änderung der Höhendifferenz zwischen zwei Punkten und unter Ermittlung der Standardabweichung einer entsprechenden Messreihe erreichen. Eine vergleichbare Formulierung existiert bereits in der USamerikanischen Richtlinie ACI117, bei der Kennzahlen für die Ebenheit des Bodens toleriert werden. Die Ermittlung der Kennzahlen wird in der Richtlinie ASTM-E-1155m [ASTM-1155] beschrieben und beruht auf der statistischen Standardabweichung einer Messreihe.

Ein mit der ASTM-E-1155m vergleichbares Bewertungssystem zeigt sowohl die Eigenschaft, mit dem Unebenheitsmaß zu korrelieren, als auch den Effekt, die Welligkeit eines Bodens mit zu berücksichtigen. In diesem Bewertungssystem wird für die aus der Standardabweichung gebildete Kennzahl eine Grenze definiert, bis zu der ein Boden für den Betrieb eines Flurförderzeugs geeignet ist.

Zusätzlich ist eine Absicherung der Bodenqualität gegen zu große Absolutauslenkungen durch Definition einer begrenzenden Höhendifferenz quer zur Fahrspur in Anlehnung an die DIN 15185 T1 als zweites Kriterium sinnvoll.

Eine messtechnische und mathematische Überprüfung des Bodens nach den genanten Grundsätzen ist einfach zu realisieren und wird im Anwendungsbereich der ACI117 bereits praktiziert.

Somit erfüllt das geschilderte Konzept sämtliche Anforderungen an eine ideale Tauglichkeitsprüfung für Böden in von Flurförderzeugen bedienten Lägern. 


\section{Literatur}

[Braun1991]

[Günthner2005]

[Kortüm1994]

[Mitschke1989]

[Popp1993]

[Schuknecht91]

[ACI-117]

[ASTM-1155]

[DIN-15185]

[DIN-18202]
Braun, H., Hellenbroich, T.: Messergebnisse von Straßenunebenheiten. VDIBerichte, 877, S. 47-80 Düsseldorf: VDI-Verlag, 1991

Günthner, W.; Schmalzl, J.; Lagerbodenqualität und Laufruhe. IN: Hebezeuge und Fördermittel (2005) 4, S. 188 - 190

Kortüm, W.; Lugner, P.: Systemdynamik und Regelung von Fahrzeugen. Berlin, Heidelberg: Springer, 1994

Mitschke, M.; Dynamik der Kraftfahrzeuge. Band B: Schwingungen, Berlin, Heidelberg: Springer, 1989

Popp, K.; Schielen, W.; Fahrzeugdynamik. Stuttgart: Teubner 1993

Schuknecht, F., et al.: Messung von Nutzfahrzeugschwingungen auf der Straße Nachbildung der Fahrbahnunebenheiten am Prüfstand und Berechnungsverfahren für die Computersimulation. VDI-Berichte Nr. 877, S. 119-134 Düsseldorf: VDI-Verlag, 1991

ACI-117: Specifications for Tolerances for Concrete Construction and Materials. 2004

ASTM E 1155M: Standard Test Method for Determining FF Floor Flatness and FL Floor Levelness Numbers [Metric]. 2001.

DIN 15185 Teil 1: Lagersysteme mit leitliniengeführten Flurförderzeugen Anforderung an Boden, Regal und sonstige Anforderungen. August 1991 Toleranzen im Hochbau - Bauwerke. April 1997 